\title{
Bright solitary waves of atomic Bose-Einstein condensates under rotation
}

\author{
N. A. Jamaludin, ${ }^{1}$ N. G. Parker, ${ }^{1,2}$ and A. M. Martin ${ }^{1}$ \\ ${ }^{1}$ School of Physics, University of Melbourne, Parkville, Victoria 3010, Australia \\ ${ }^{2}$ Department of Physics and Astronomy, McMaster University, Hamilton, Ontario, Canada L8S 4M1
}

(Received 2 March 2008; published 29 May 2008)

\begin{abstract}
We analyze the rotation of bright solitary waves formed of atomic Bose-Einstein condensates with attractive atomic interactions. By employing a variational technique and assuming an irrotational quadrupolar flow field, we map out the variational solutions in the rotating frame. In particular, we show that rotation has a considerable stabilizing effect on the system, significantly raising the critical threshold for collapse of the bright solitary waves.
\end{abstract}

DOI: $10.1103 /$ PhysRevA.77.051603

PACS number(s): 03.75.Kk, 34.20.Cf, 47.20.-k

In recent years, bright solitary waves (BSWs) have been created using ultracold atomic Bose-Einstein condensates (BECs) [1]. For attractive atomic interactions, these matter waves are self-trapped in the longitudinal direction [2-6] and are analogous to one-dimensional (1D) solitons [7]. However, these states must be confined in the remaining directions by a waveguide and can retain three-dimensional effects. The most lucid example is the collapse instability: in 3D, a homogeneous, untrapped BEC with attractive interactions is unstable to collapse [8] while the 1D limit is stable to collapse. The presence of external trapping stabilizes the BEC up to a critical atom number (or interaction strength) before collapse is triggered $[9,10]$. The collapse instability has limited BSW experiments to only a few thousand atoms per BSW [1]. The BSW solutions, and their critical points, have been studied theoretically [3-6], with variational approaches shown to give very good predictions. BSWs offer exciting prospects for atom-surface detection [11], and their collisions, which show intriguing behavior and may have applications in interferometry, are also prone to collapse instabilities [5,6,13-15]. As such, it is pertinent to consider approaches to suppress collapse in attractive BECs. Periodic modulation of the interaction strength, made possible by employing a Feshbach resonance, is predicted to partially stabilize against collapse [16], although when the interaction is sign-definite and the mean is attractive, collapse is inevitable [17]. The presence of a quantized vortex is also predicted to raise the threshold for collapse [13,18-20], although the presence of a vortex in an attractive condensate under harmonic trapping is energetically unstable [18,21]. Recent work also considered the possibility of fragmented states, where the occupation of more than one mode [22] is postulated, such that the critical number for collapse increases.

The rotation of repulsive BECs has been considered extensively experimentally and theoretically (see [23] for a review). One method is to mechanically rotate the BEC in an elliptical trap [24-26] formed by time-dependent laser or magnetic fields. At low rotation frequency $\Omega$, the condensate remains irrotational and vortex-free. According to a hydrodynamical model, the BEC can access a family of rotating stationary solutions, characterized by a quadrupolar irrotational flow pattern [27-32] and confirmed experimentally [25]. At a critical rotation frequency, when these irrotational solutions become unstable [30,32], vortices are nucleated and form a vortex lattice. In the context of attractive BECs, theoretical work has shown that a center-of-mass mode is favored under rotation rather than the occurrence of vortices $[21,33]$. Under harmonic trapping, this mode becomes excited when $\Omega$ exceeds the trap frequency. In the context of rotating optical lattices, solitons and vortex solitons have also been studied theoretically [34].

In this work, we consider the rotation of BSWs in an elliptical trap about the longitudinal axis. We employ a variational technique based on assuming an ansatz for the BSW profile, which incorporates a quadrupolar irrotational flow pattern. By deriving the variational energy of the system, we obtain the BSW solutions and analyze their response to rotation, finding that rotation can significantly increase the critical point for collapse.

We consider a BEC confined by an atomic "waveguide" potential, under rotation at frequency $\Omega$ about the longitudinal axis. In the limit of zero temperature, the BEC can be described by a mean-field "wave function" $\Psi(\mathbf{r}, t)$ that satisfies the Gross-Pitaevskii equation (GPE) [23],

$$
i \hbar \frac{\partial \Psi}{\partial t}=\left[-\frac{\hbar^{2}}{2 m} \nabla^{2}+V(\mathbf{r})+g|\Psi|^{2}-\Omega \hat{L}_{z}\right] \Psi,
$$

where $m$ is the atomic mass and $g=4 \pi \hbar^{2} a_{\mathrm{s}} / m$ parametrizes the atomic interactions, with $a_{\mathrm{s}}$ being the $s$-wave scattering length. The $\Omega \hat{L}_{z}$ term accounts for frame rotation, where $\hat{L}_{z}$ $=-i \hbar\left(x \frac{\partial}{\partial y}-y \frac{\partial}{\partial x}\right)$ is the $z$-component angular momentum operator. We consider a harmonic trapping potential,

$$
V(\mathbf{r})=\frac{1}{2} m \omega_{r}^{2}\left[(1-\epsilon) x^{2}+(1+\epsilon) y^{2}+\lambda^{2} z^{2}\right],
$$

where $\omega_{r}$ is the average trap frequency in the $x-y$ plane, $\epsilon$ is the trap ellipticity in the $x-y$ plane, and $\lambda=\omega_{z} / \omega_{r}$ is the trap ratio that determines the axial trap strength.

To study BSW solutions, we employ a variational technique. This involves assuming a BSW ansatz and minimizing its energy to obtain variational solutions. This technique has been employed for nonrotating BSWs and has been shown to give very good agreement with the full solution of the GPE. As we will see, the rotating frame solutions can be mapped onto nonrotating solutions with reduced trap frequencies (due to centrifugal effects), and as such we expect the variational technique to continue being an excellent description. 
For weak axial trapping $(\lambda \ll 1)$, a sech ansatz for the BSW is employed,

$$
\Psi_{S}=\sqrt{\frac{N}{2 l_{x} l_{y} l_{z} \pi}} e^{-x^{2} / 2 l_{x}^{2}} e^{-y^{2} / 2 l_{y}^{2} \operatorname{sech}\left(\frac{z}{l_{z}}\right) e^{i \alpha x y},}
$$

where $N$ is the atom number. For $\alpha=0$, this ansatz is identical to that used in Refs. [4-6], with the sech axial profile appropriate because it is the form of the 1D soliton solution [7]. The term $e^{i \alpha x y}$ introduces a quadrupolar flow pattern to the BEC, of amplitude $\alpha$. This preserves irrotationality and has been successful in modeling vortex-free repulsive BECs under rotation [25,28-32].

The total energy of the system is defined by

$$
\begin{aligned}
E= & \int\left[\frac{\hbar^{2}}{2 m}|\nabla \Psi|^{2}+V(\mathbf{r})|\Psi|^{2}+\frac{g}{2}|\Psi|^{4}\right. \\
& \left.+i \hbar \Omega\left(\Psi^{\star} x \frac{\partial \Psi}{\partial y}-\Psi y \frac{\partial \Psi^{\star}}{\partial x}\right)\right] d^{3} \mathbf{r} .
\end{aligned}
$$

Insertion of the ansatz into Eq. (4) gives the energy $E_{S}$,

$$
\begin{aligned}
\frac{E_{S}}{N}= & \frac{\hbar^{2}}{2 m}\left[\frac{1}{2 l_{x}^{2}}+\frac{1}{2 l_{y}^{2}}+\frac{1}{3 l_{z}^{2}}+\frac{l_{x}^{2} \alpha^{2}}{2}+\frac{l_{y}^{2} \alpha^{2}}{2}\right] \\
& +\frac{m \omega_{r}^{2}}{4}\left[(1-\epsilon) l_{x}^{2}+(1+\epsilon) l_{y}^{2}+\frac{\lambda^{2} \pi^{2}}{6} l_{z}^{2}\right] \\
& +\frac{g N}{4 \pi l_{x} l_{y} l_{z}}-\frac{\hbar \Omega_{z} \alpha}{2}\left(l_{x}^{2}+l_{y}^{2}\right) .
\end{aligned}
$$

We can reduce the number of variables in Eq. (5) as follows. Under the hydrodynamical interpretation, the meanfield wave function can be expressed as $\Psi(\mathbf{r}, t)$ $=\sqrt{n}(\mathbf{r}, t) \exp [i \phi(\mathbf{r}, t)]$, where $n(\mathbf{r}, t)$ and $\phi(\mathbf{r}, t)$ are the condensate density and phase, respectively. Furthermore, $\mathbf{v}(\mathbf{r}, t)=(\hbar / m) \boldsymbol{\nabla} \phi(\mathbf{r}, t)$ is the "fluid" velocity. Inserting this into the GPE and equating imaginary parts, one derives a continuity equation given by

$$
\frac{\partial n}{\partial t}+\nabla \cdot(n[\mathbf{v}-\Omega \times \mathbf{r}])=0 .
$$

Our irrotational phase distribution $\phi(\mathbf{r})=\alpha x y$ corresponds to a velocity distribution $\mathbf{v}(\mathbf{r})=\hbar \alpha(y \hat{\mathbf{i}}+x \hat{\mathbf{j}}) / m$. Inserting this into the continuity equation and setting $\partial n / \partial t=0$, we find that stationary solutions satisfy

$$
\alpha= \pm \frac{m \Omega_{z}}{\hbar}\left(\frac{l_{x}^{2}-l_{y}^{2}}{l_{x}^{2}+l_{y}^{2}}\right) .
$$

Note that $\alpha$ can be positive or negative, resulting in two "branches" of solutions. For $\alpha>0$, the BSW is wider in the $x$ direction than in the $y$ direction, and vice versa for $\alpha<0$. We can thus eliminate $\alpha$ from Eq. (5). For simplicity, we employ rescaled variables $\gamma_{x}=l_{x} / a_{r}, \quad \gamma_{y}=l_{y} / a_{r}, \quad \gamma_{z}=l_{z} / a_{r}$, $\widetilde{\Omega}=\Omega / \omega_{r}$, and $\varepsilon_{S}=E_{S} /\left(N \hbar \omega_{r}\right)$, where $a_{r}=\sqrt{\hbar / m \omega_{r}}$ is the radial harmonic-oscillator length. Introducing the dimensionless interaction parameter $k=N\left|a_{\mathrm{s}}\right| / a_{r}$, the ansatz energy becomes
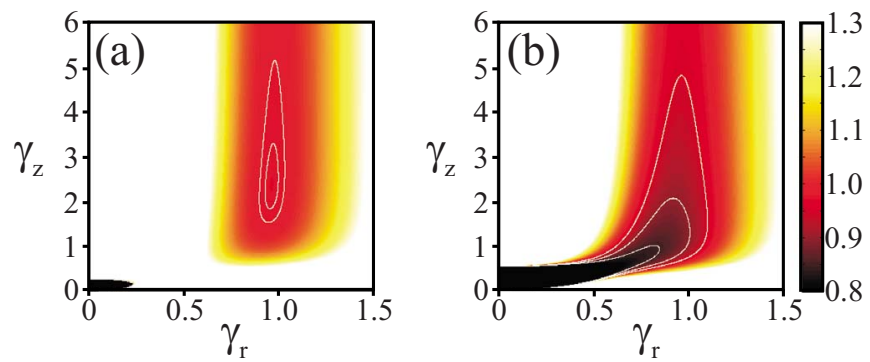

FIG. 1. (Color online) Energy landscape of the nonrotating and nonelliptical system for $\lambda=0$ according to Eq. (8). (a) Stable regime $k=0.4<k_{c}$ featuring a local-energy minimum, i.e., the BSW solution. (b) Unstable regime $k=0.8>k_{c}$, where the whole parameter space is unstable to collapse. White contours highlight the shape of the landscapes. Since Eq. (8) is cylindrically symmetric in this case, we introduce a radial length scale $\gamma_{r}^{2}=\left(\gamma_{x}^{2}+\gamma_{y}^{2}\right) / 4$.

$$
\begin{aligned}
\varepsilon_{S}= & \frac{1}{4}\left[\frac{1}{\gamma_{x}^{2}}+\frac{1}{\gamma_{y}^{2}}+\frac{2}{3 \gamma_{z}^{2}}+(1-\epsilon) \gamma_{x}^{2}+(1+\epsilon) \gamma_{y}^{2}+\frac{\lambda^{2} \pi^{2}}{6} \gamma_{z}^{2}\right] \\
& -\frac{k}{3 \gamma_{x} \gamma_{y} \gamma_{z}} \pm \frac{\widetilde{\Omega}_{z}^{2}}{4}\left[\gamma_{x}^{2}-\gamma_{y}^{2}\right]\left[\frac{\gamma_{x}^{2}-\gamma_{y}^{2}}{\gamma_{x}^{2}+\gamma_{y}^{2}}-2\right]
\end{aligned}
$$

Equation (8) is valid for $\lambda \ll 1$. Under tight axial trapping $\lambda \gg 0$, this direction is dominated by the trap rather than the interactions and it is appropriate to consider a Gaussian ansatz,

$$
\Psi_{G}=\sqrt{\frac{N}{l_{x} l_{y} l_{z} \pi^{3 / 2}}} e^{-x^{2} / 2 l_{x}^{2}} e^{-y^{2} / 2 l_{y}^{2}} e^{-z^{2} / 2 l_{z}^{2}} e^{i \alpha x y} .
$$

The rescaled energy for the Gaussian ansatz is then

$$
\begin{aligned}
\varepsilon_{G}= & \frac{1}{4}\left[\frac{1}{\gamma_{x}^{2}}+\frac{1}{\gamma_{y}^{2}}+\frac{1}{\gamma_{z}^{2}}+(1-\epsilon) \gamma_{x}^{2}+(1+\epsilon) \gamma_{y}^{2}+\lambda^{2} \gamma_{z}^{2}\right] \\
& -\frac{k}{\sqrt{2 \pi} \gamma_{x} \gamma_{y} \gamma_{z}} \pm \frac{\widetilde{\Omega}_{z}^{2}}{4}\left[\gamma_{x}^{2}-\gamma_{y}^{2}\right]\left[\frac{\gamma_{x}^{2}-\gamma_{y}^{2}}{\gamma_{x}^{2}+\gamma_{y}^{2}}-2\right] .
\end{aligned}
$$

For the regimes of interest, the sech and Gaussian ansatz give similar results, typically differing by less than $10 \%$ [6]. The 3D energy landscapes of the rotating BSWs as a function of the length scales $\gamma_{x}, \gamma_{y}$, and $\gamma_{z}$ can be mapped out, with variational BSW solutions existing where there is a local-energy minimum. The BSW has widths $\gamma_{x}^{0}, \gamma_{y}^{0}$, and $\gamma_{z}^{0}$, energy $\varepsilon_{0}$, and quadrupolar flow amplitude $\alpha_{0}$.

We first revisit the $\lambda=0$ BSW solutions in the absence of rotation and ellipticity, as studied previously using the $\alpha=0$ limits of Eq. (8) [4,6] and Eq. (10) [2]. Here the energy landscape is cylindrically symmetric $(\epsilon=0)$ and so we introduce a radial length scale $\gamma_{r}^{2}=\left(\gamma_{x}^{2}+\gamma_{y}^{2}\right) / 4$. A typical energy landscape, according to Eq. (8), for a stable BSW solution $(k=0.4)$ is shown in Fig. 1(a). At the origin, the interaction term in Eq. (8) diverges to negative values and is a region of collapse of the BSW. However, there exists a local-energy minimum that represents the self-trapped BSW solution. A typical unstable energy landscape $(k=0.8)$ is shown in Fig. 1(b). No local energy minimum exists, and the system is unstable to collapse. The critical interaction strength for col- 

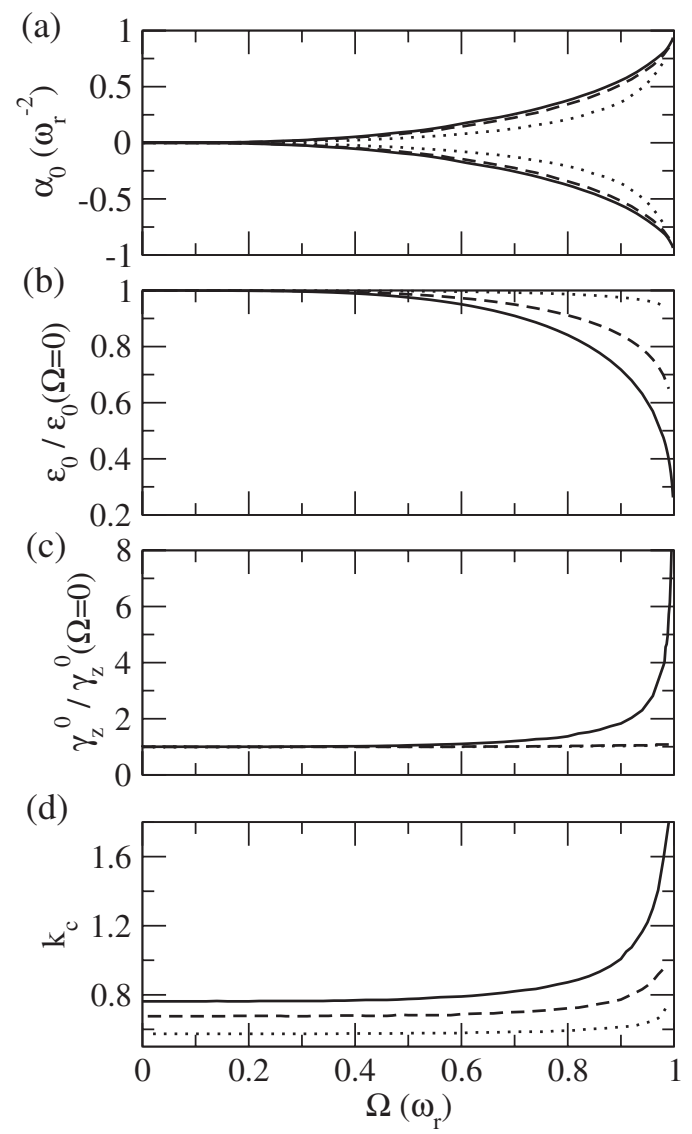

FIG. 2. Properties of $\epsilon=0$ BSW solutions as a function of $\Omega$ for $\lambda=0$ (solid line) and the axially trapped cases of $\lambda=1$ (dashed lines) and 5 (dotted lines). (a) Quadrupolar flow amplitude $\alpha_{0}$ for interaction parameter $k=0.4$. (b) BSW energy $\varepsilon_{0}$ for $k=0.4$. (c) Axial length scale $\gamma_{z}^{0}$ for $k=0.4$. (d) Critical interaction parameter for collapse $k_{c}$. Note that for $\lambda=0(\lambda>0)$ we employ the sech (Gaussian) ansatz.

lapse is determined to be $k_{c}=0.76$, in good agreement with the full solution of the GPE, which gives $k_{c} \approx 0.68[4,6]$.

Figures 2(a)-2(c) show how the key parameters vary as rotation is introduced for a fixed interaction parameter $k$ $=0.4$ and $\epsilon=0$. For $\Omega>0$, the symmetry between $\gamma_{x}$ and $\gamma_{y}$ is broken and the solutions have nonzero $\alpha_{0}$ [Fig. 2(a)]. We see the formation of two branches of $\alpha_{0}$. Due to the trap symmetry in the $x-y$ plane, the branches are symmetric about the $\alpha_{0}=0$ axis, with the upper branch being elongated in the $x$ direction and the lower branch being elongated in the $y$ direction. As $\Omega$ increases, so too does the magnitude of $\alpha_{0}$, implying a spreading of the BSW in the $x-y$ plane. This is because of the growth of an outward centrifugal force. As $\Omega$ approaches $\omega_{r}, \alpha_{0}$ diverges to $\pm \infty$. This is because, at $\Omega$ $=\omega_{r}$, the centrifugal force exactly balances the trapping potential, and the BEC is untrapped in the $x-y$ plane. Since the BEC center of mass becomes dynamically unstable, this is termed the center-of-mass instability [35]. The BSW energy [Fig. 2(b)] decreases toward zero as $\Omega \rightarrow \omega_{r}$ as a result of the reduced density. The axial length scale [Fig. 2(c)] grows with $\Omega$ since the radial spreading dilutes the interaction strength and forms a less tightly bound BSW.

We have isolated the critical interaction parameter for col-
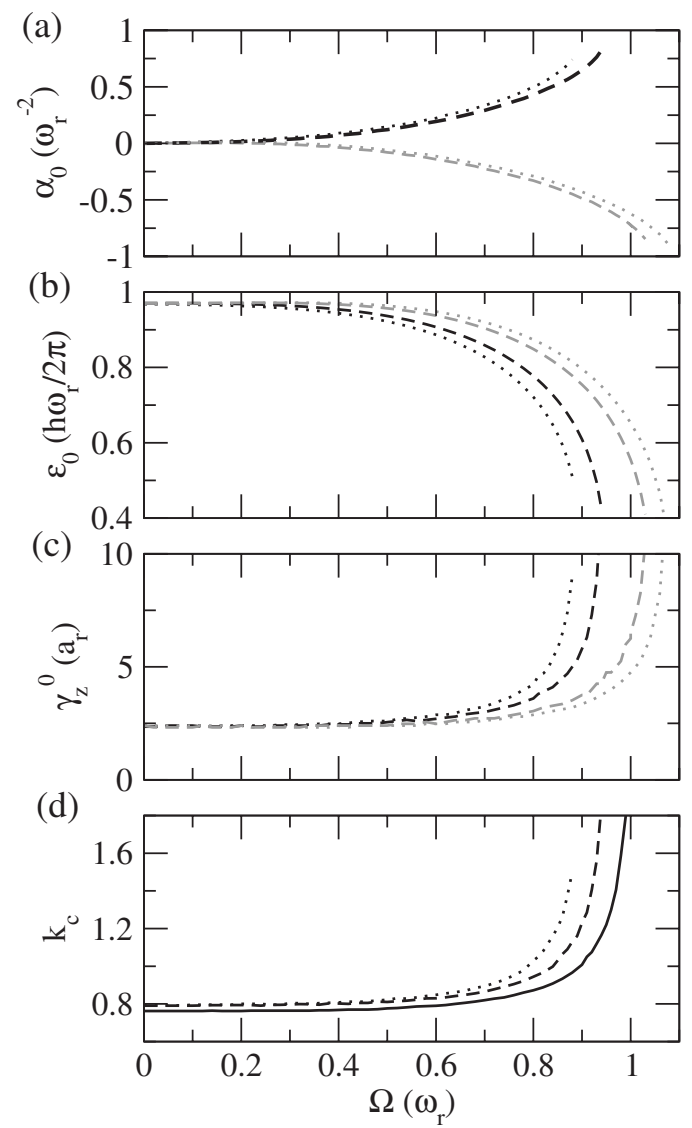

FIG. 3. Properties of the rotating $\lambda=0$ BSWs for elliptical traps of $\epsilon=0.1$ (dashed lines) and 0.2 (dotted lines). Black (gray) lines indicate upper (lower) branch solutions. (a) Quadrupolar flow amplitude $\alpha_{0}$ for $k=0.4$. (b) BSW energies $\varepsilon_{0}$. (c) Axial length scales $\gamma_{z}^{0}$. (d) Critical interaction parameter for collapse $k_{c}$ of the upper branch solutions.

lapse $k_{c}$ as a function of $\Omega$ with the results shown in Fig. 2(d). The most striking feature is that $k_{c}$ dramatically increases as $\Omega_{z}$ approaches $\omega_{r}$. This is directly associated with the radial spreading and reduced density of the rotating solutions. Specifically, for $\Omega / \omega_{r}=0.9, k_{c}$ is approximately $50 \%$ larger than its nonrotating value while for $\Omega / \omega_{r}=0.97, k_{c}$ is approximately twice as large.

In Fig. 2, we also consider the presence of significant axial trapping $\lambda=1$ (dashed line) and 5 (dotted line), for which we employ the Gaussian ansatz. We see similar qualitative behavior to the $\lambda=0$ case: a divergent growth of $\alpha_{0}$ [Fig. 2(a)] and a decrease in $\varepsilon_{0}$ [Fig. 2(b)]. However, the magnitudes are consistently less than the corresponding $\lambda$ $=0$ results. The axial length scales [Fig. 2(c)] show little variation with $\Omega$ since this is now dominated by the external axial trapping. The critical point for collapse $k_{c}$ grows with $\Omega$, but at a slower rate than for $\lambda=0$, with axial trapping reducing $k_{c}[6,12]$.

The $\epsilon=0$ limit is somewhat unphysical since no torque is actually applied to the BEC. We now consider the more realistic case of finite trap ellipticity. The results for $\lambda=0$ BSWs under $\epsilon=0.1$ and 0.2 are presented in Fig. 3. The finite ellipticity breaks the symmetry in the $x-y$ plane and therefore in the branches of $\alpha_{0}$. The upper branch solutions are elon- 
gated in the $x$ direction, which has trap frequency $\omega_{x}$ $=\sqrt{(1-\epsilon)} \omega_{r}$. The center-of-mass instability then occurs when $\Omega=\sqrt{(1-\epsilon)} \omega_{r}$, which is why the divergence in $\alpha_{0}$ shifts to lower $\Omega$. Conversely, the lower branch solutions diverge at $\Omega=\sqrt{1+\epsilon} \omega_{r}$ and so become shifted toward larger $\Omega$.

The energy [Fig. 3(b)] shows that the upper branch solutions have lower energy. That is, it is energetically favorable for the BSW to be elongated in $x$ rather than $y$, since the trap is weaker in this direction. Consequently, only the upper branch solutions would ever be observed. Apart from the shift in the asymptotes introduced by the finite ellipticity, the behavior of $\alpha_{0}, \epsilon_{0}$, and $\lambda_{z}^{0}$ is qualitatively similar to the $\epsilon$ $=0$ case. In Fig. 2(d), we plot the critical interaction parameter for collapse $k_{c}$ of the upper branch solutions. As for the $\epsilon=0$ case, a dramatic in increase in $k_{c}$ is predicted as $\Omega \rightarrow \sqrt{1-\epsilon} \omega_{r}$.

In considering static solutions (in the rotating frame), our results apply to when collapse is approached adiabatically. If collapse in induced nonadiabatically, e.g., by rapidly ramp- ing up the attractive interactions, the dynamics become dependent on the functional form of the ramping with collective mode excitation. Our methodology could be extended to describe such dynamics by deriving the equations of motion for the rotating Gaussian ansatz, as performed for the nonrotating case [2].

In this work, we have employed a variational technique to study bright solitary matter-wave solutions under rotation in elliptical traps. This is made possible by incorporating an irrotational quadrupolar flow pattern into the variational ansatz. Importantly, the BSW becomes more stable to the collapse instability when under rotation, enabling a significant increase in the number of atoms before the onset of collapse. Our study opens the door to controllably stabilize bright solitary waves against collapse, as well as the novel exploration of collapse dynamics in rotating systems.

We acknowledge funding from the ARC and the Canadian Commonwealth Scholarship Program (N.G.P.).
[1] K. E. Strecker et al., Nature 417, 150 (2002); L. Khaykovich et al., Science 296, 1290 (2002); S. L. Cornish, S. T. Thompson, and C. E. Wieman, Phys. Rev. Lett. 96, 170401 (2006).

[2] V. M. Pérez-Garcia, H. Michinel, J. I. Cirac, M. Lewenstein, and P. Zoller, Phys. Rev. A 56, 1424 (1997).

[3] V. M. Pérez-Garcia, H. Michinel, and H. Herrero, Phys. Rev. A 57, 3837 (1998).

[4] L. D. Carr and Y. Castin, Phys. Rev. A 66, 063602 (2002).

[5] L. Salasnich, A. Parola, and L. Reatto, Phys. Rev. A 66, 043603 (2002)

[6] N. G. Parker et al., J. Phys. B 40, 3127 (2007).

[7] V. E. Zakharov and A. B. Shabat, Sov. Phys. JETP 34, 62 (1972).

[8] P. Nozières and D. Pines, Theory of Quantum Liquids Vol. II (Addison-Wesley, Redwood City, CA, 1990).

[9] C. C. Bradley, C. A. Sackett, and R. G. Hulet, Phys. Rev. Lett. 78, 985 (1997).

[10] J. L. Roberts et al., Phys. Rev. Lett. 86, 4211 (2001).

[11] S. L. Cornish et al., e-print arXiv:802.4362.

[12] A. Gammal, T. Frederico, and L. Tomio, Phys. Rev. A 64, 055602 (2001); A. Gammal, L. Tomio, and T. Frederico, ibid. 66, 043619 (2002).

[13] S. K. Adhikari, New J. Phys. 5, 137 (2003).

[14] L. D. Carr and J. Brand, Phys. Rev. Lett. 92, 040401 (2004); B. B. Baizakov, B. A. Malomed, and M. Salerno, Phys. Rev. A 70, 053613 (2004).

[15] N. G. Parker, A. M. Martin, S. L. Cornish, and C. S. Adams, J. Phys. B 41, 045303 (2008).

[16] H. Saito and M. Ueda, Phys. Rev. Lett. 90, 040403 (2003); F. K. Abdullaev, J. G. Caputo, R. A. Kraenkel, and B. A. Malomed, Phys. Rev. A 67, 013605 (2003); S. K. Adhikari, ibid. 69, 063613 (2004); G. D. Montesinos, V. M. Perez-Garcia, and P. J. Torres, Physica D 191, 193 (2004); A. Itin, T. Morishita, and S. Watanabe, Phys. Rev. A 74, 033613 (2006).

[17] V. V. Konotop and P. Pacciani, Phys. Rev. Lett. 94, 240405 (2005).
[18] F. Dalfovo and S. Stringari, Phys. Rev. A 53, 2477 (1996)

[19] S. K. Adhikari, Phys. Rev. E 65, 016703 (2001).

[20] S. K. Adhikari, Phys. Rev. A 66, 043601 (2002).

[21] N. K. Wilkin, J. M. F. Gunn, and R. A. Smith, Phys. Rev. Lett. 80, 2265 (1998).

[22] L. S. Cederbaum, A. I. Streltsov, and O. E. Alon, Phys. Rev. Lett. 100, 040402 (2008).

[23] Emergent Nonlinear Phenomena in Bose-Einstein Condensates: Theory and Experiment, edited by P. G. Kevrekidis, D. J. Frantzeskakis, and R. Carretero-Gonzalez, (Springer, Berlin, 2008).

[24] K. W. Madison, F. Chevy, W. Wohlleben, and J. Dalibard, Phys. Rev. Lett. 84, 806 (2000).

[25] K. W. Madison, F. Chevy, V. Bretin, and J. Dalibard, Phys. Rev. Lett. 86, 4443 (2001).

[26] E. Hodby, G. Hechenblaikner, S. A. Hopkins, O. M. Marago, and C. J. Foot, Phys. Rev. Lett. 88, 010405 (2001).

[27] Y. Castin and R. Dum, Eur. Phys. J. D 7, 399 (1999).

[28] A. Recati, F. Zambelli, and S. Stringari, Phys. Rev. Lett. 86, 377 (2001).

[29] S. Sinha and Y. Castin, Phys. Rev. Lett. 87, 190402 (2001).

[30] N. G. Parker, R. M. W. van Bijnen, and A. M. Martin, Phys. Rev. A 73, 061603(R) (2006).

[31] R. M. W. van Bijnen, D. H. J. O’Dell, N. G. Parker, and A. M. Martin, Phys. Rev. Lett. 98, 150401 (2007).

[32] I. Corro, N. G. Parker, and A. M. Martin, J. Phys. B 40, 3615 (2007).

[33] A. Collin, E. Lundh, and K.-A. Suominen, Phys. Rev. A 71, 023613 (2005); A. Collin, ibid. 73, 013611 (2006).

[34] H. Sakaguchi and B. A. Malomed, Phys. Rev. A 75, 013609 (2007); Y. V. Kartashov, B. A. Malomed, and L. Torner, ibid. 75, 061602(R) (2007); J. Cuevas, B. A. Malomed, and P. G. Kevrekidis, Phys. Rev. E 76, 046608 (2007).

[35] P. Rosenbusch et al., Phys. Rev. Lett. 88, 250403 (2002); N. G. Parker and C. S. Adams, J. Phys. B 39, 43 (2006). 